\title{
Cataract, CTCAE 5.0
}

National Cancer Institute

\section{Source}

National Cancer Institute. Cataract, CT CAE 5.0. NCI Thesaurus. Code C146674.

A disorder characterized by partial or complete opacity of the crystalline lens of one or both eyes. This results in a decrease in visual acuity and eventual blindness if untreated. 